\title{
Fabrication of pH-electroactive Bacterial Cellulose/Polyaniline Hydrogel for the Development of a Controlled Drug Release System
}

\author{
Sixiang Li,${ }^{1 *}$ Ashwak Jasim, ${ }^{1 *}$ Weiwei Zhao, ${ }^{2}$ Lina Fu, ${ }^{3}$ Muhammad Wajid Ullah, ${ }^{1 *}$ Zhijun Shi, ${ }^{1 *}$ and Guang Yang ${ }^{1 *}$
}

In the current study, a pH-electroactive hydrogel of bacterial cellulose (BC) and polyaniline (PAni) was fabricated through chemical oxidation polymerization for the development of a controlled drug release system. PAni was densely arrayed along the BC fibers and berberine hydrochloride $(\mathrm{BH})$ was diffused within the hydrogel matrix as confirmed by field emission scanning electron microscope (FE$\mathrm{SEM}$ ) and fourier transform infrared (FTIR) analyses. The $\mathrm{pH}$-electroactive drug release behavior of BC/PAni hydrogel was investigated in vitro. Variation in $\mathrm{pH}$ values from 2.2 to 11 demonstrated different drug release profiles from BC/PAni electroactive hydrogel: fast in alkaline and slow in acidic environments as indicated by a color change between green and dark, respectively. The drug release from $\mathrm{BC} / \mathrm{PAni}$ hydrogel was stable and continuous which showed a typical $\mathrm{pH}$-electroactive drug release behavior. The drug release curves closely matched the Korsemyer-Peppas kinetic model with free diffusion. Owing to its porous nature, conductive properties, and pHelectroactive drug release behavior, the developed BC/PAni hydrogel system can find potential applications as a controlled drug release system.

Keywords: Bacterial cellulose; Polyaniline; pH-electroactive hydrogel; Controlled drug release

Received 3 September 2018, Accepted 10 October 2018

DOI: $10.30919 /$ esmm $5 f 120$

\section{Introduction}

Different strategies developed for controlled and targeted drug delivery over the past few decades have received immense consideration in research and clinical applications. ${ }^{1-3}$ Nevertheless, the development of responsive and controlled drug delivery system for active compounds is still a major challenge; therefore, various drug delivery systems have been studied by applying external stimuli such as electric and magnetic fields, ultrasonic waves, light, pressure, and $\mathrm{pH}$ for drug release behaviors in a premeditated and controlled fashion. To this end, the electro-stimulated drug release devices (EDRDs) are specially designed to ensure a programmed drug release profile controlled by applying an external voltage or current. ${ }^{4}$

Currently, the design and use of various functional biomaterials for drug loading and release in the development of EDRDs have become a promising trend. The conducting polymers are famous for

${ }^{1}$ Department of Biomedical Engineering, College of Life Science and Technology, Huazhong University of Science and Technology, Wuhan 430074, PR China

${ }^{2}$ School of Mechanical and Electronic Engineering, Wuhan University of Technology, Wuhan 430070, PR China

${ }^{3}$ Department of Surgery-Transplant and Mary \& Dick Holland Regenerative Medicine Program, University of Nebraska Medical Center, Omaha, NE 68198, USA

*E-mail:wajid_kundi@hust.edu.cn; shizhijun@hust.edu.cn;

yang_sunny@yahoo.com. their applications in the development of supercapacitors, which perform as good as metallic oxides ${ }^{5,6}$ and simultaneously possessing good biocompatibility. Therefore, the use of different conducting polymers is receiving immense consideration in the development of EDRDs owing to their potentials to change their redox state upon electrical stimulation, which in turn modulates the delivery, exchange, and the movement of specific ions and molecules in a programmed manner. In one such study, the drug release from an ion gate membrane of polypyrrole (PPy) was controlled by single and stepwise controlled electrical stimulation; ${ }^{7}$ however, the amount of drug released was too low for practical therapeutic applications. Therefore, the conducting polymers can be polymerized within the matrix of a polymer with different pore sizes for improved drug loading and release. Lira et al. synthesized a semi-interpenetrating network of polyacrylamide and polyaniline (PAni) that allowed the storage of a large amount of molecules and their release in an electrochemically controlled fashion. ${ }^{8}$ In another study, the surface of a gold electrode was coated with the fibers of a biodegradable polymer, poly (lactic-co-glycolic acid) (PLGA), via electrospinning and poly (3,4-ethylenedioxythiophene) (PEDOT) nanotubes by electrochemical deposition. The PLGA improved the drug loading capacity while PEDOT nanotubes decreased the impedance and increased the charge capacity of gold electrode. As a result, the release of dexamethasone was precisely controlled via external electrical stimulation. ${ }^{9}$

Bacterial cellulose $(\mathrm{BC})$, a natural polymer hydrogel produced by certain microbial strains ${ }^{10,11}$ and a cell-free system ${ }^{12}$ from different carbon sources, ${ }^{13}$ possesses high purity, excellent mechanical properties, high crystallinity, ultrafine fiber network, high water holding capacity and slow water loss rate, biocompatibility, and 
moldability into three dimensional structure. ${ }^{14}$ These features make $\mathrm{BC}$ an ideal material for its broad spectrum applications in regenerative medicines and tissue engineering applications, such as wounding dressing, ${ }^{15,16}$ vascular grafts, ${ }^{17}$ artificial intervertebral disc, ${ }^{18}$ biosensors, ${ }^{19,20}$ and various biomedical and electroconductive devices and applications. ${ }^{21}$ In our previous study, BC was used as a drug carrier where its fine network was manipulated to maintain the drug concentration in a desired therapeutic range that resulted in extended drug release time..$^{22}$ In another study, BC was used as a hydrogel matrix and combined with conductive polymers, PAni and PPy, to fabricate biphasic electroactive hydrogels with integrated electroactivity and biocompatibility. ${ }^{23}$

In the current study, a controlled drug release system based on biocompatible $\mathrm{BC}$ and electroactive PAni was developed. The multilayered BC/PAni showed a sandwiched structure with different porosity inside and outside the hydrogel after a controlled chemical oxidation polymerization reaction. The BC/PAni hydrogel was loaded with a drug berberine hydrochloride $(\mathrm{BH})$, the main constituent of the Chinese medicinal herbs Rhizoma coptidis (Chinese name: Huang lian) and Cortex phellodendri (Chinese name: Huang bai), and evaluated for its ability to stimulate drug release under different $\mathrm{pH}$ conditions and electrical stimulation because it is poorly absorbed by oral administration and prohibited through intravenous injection. It was hypothesized that the different porosity of hydrogel inside and outside the hydrogel will allow a controlled and extended drug release under the effect of varying $\mathrm{pH}$ and electrical stimulation. The BC/PAni composite was prepared by chemical oxidation polymerization of aniline along $\mathrm{BC}$ fibers followed by impregnating $\mathrm{BH}$ into its matrix. The developed composite hydrogel showed a "sandwich" structure consisted of two external layers of $\mathrm{BC}$ and PAni which showed a good electrochemical property. The morphological changes occurred during the oxidation polymerization process were monitored by field emission scanning electron microscopy (FE-SEM) and fourier transform infrared (FTIR) spectroscopy. The drug release from the composite hydrogel was monitored under different $\mathrm{pH}$ and current flow. The developed multilayered BC/PAni sandwiched hydrogel can find potential applications in the development of a controlled drug system due to its different porous nature inside and outside of hydrogel, $\mathrm{pH}$ and electro-sensitivity, and conductive properties.

\section{Materials and Methods}

\subsection{Materials}

The chemical reagents including glucose, yeast extract, peptone, disodiumphosphate, citric acid, phosphate buffer saline (PBS), and berberine hydrochloride were purchased from Sigma-Aldrich (St. Louis, MO, USA). Concentrated hydrochloric acid, sodium hydroxide, aniline, and ammonium peroxydisulfate (APS) $\left[\left(\mathrm{NH}_{4}\right)_{2} \mathrm{~S}_{2} \mathrm{O}_{8}\right]$ of analytical grade were purchased from Sinopharm Chemical Regents Co., Ltd. (Shanghai, China). All reagents were used without further processing.

\subsection{Production of $\mathrm{BC}$ hydrogels}

The BC hydrogels were produced by Gluconacetobacter xylinum (ATCC53582) and purified according to a previously described method. ${ }^{24}$ Briefly, G. xylinum was cultured in a Hestrin and Schramm (HS) medium (pH 5.0) containing glucose $20 \mathrm{~g} / \mathrm{L}$, yeast extract 5 $\mathrm{g} / \mathrm{L}$, peptone $5 \mathrm{~g} / \mathrm{L}$, disodium phosphate $2.7 \mathrm{~g} / \mathrm{L}$, and citric acid 1.5 $\mathrm{g} / \mathrm{L}$ and incubated statically for 10 days at $30^{\circ} \mathrm{C}$. The $\mathrm{BC}$ hydrogel produced at the air-medium interface was harvested and dipped in distilled water for 2 days to remove excess medium residuals. Thereafter, it was treated with $0.3 \mathrm{~N} \mathrm{NaOH}$ at $121^{\circ} \mathrm{C}$ for $15 \mathrm{~min}$ to disrupt and dissolve any living cells and cell debris. Afterwards, the $\mathrm{BC}$ hydrogel was purified by washing several times with distilled water until its $\mathrm{pH}$ become neutral and stored at $4{ }^{\circ} \mathrm{C}$ for further use.

\subsection{Preparation of $\mathrm{BC} /$ PAni composite hydrogels}

$\mathrm{BC} / \mathrm{PAni}$ hydrogels were prepared according to a previously reported method. ${ }^{25}$ Briefly, the BC hydrogels were immersed in aniline hydrochloride solution prepared by mixing $0.02 \mathrm{M}$ aniline and 0.06 $\mathrm{M} \mathrm{HCl} \mathrm{in} 100 \mathrm{~mL}$ distilled water and incubated at ambient temperature for $24 \mathrm{~h}$ to allow the infiltration of aniline into the $\mathrm{BC}$ hydrogel matrix. Thereafter, the $\mathrm{BC}$ hydrogels were dipped in 100 $\mathrm{mL}$ of $0.2 \mathrm{~mol} / \mathrm{L}$ APS solution for different time intervals at room temperature to allow loading and in situ polymerization of PAni with $\mathrm{BC}$ fibers. The BC/PAni composites were rinsed several times with distilled water to remove APS and accessory substance until its $\mathrm{pH}$ become neutral. The sandwiched structure of BC/PAni was obtained by removing the edges and cutting into $1 \mathrm{~cm} 1 \mathrm{~cm}$ squares. Finally, the fabricated multilayered BC/PAni hydrogels were immersed in PBS (pH 7.4) and stored in $4^{\circ} \mathrm{C}$.

\subsection{Drug loading in electroactive BC/PAni hydrogels}

The model drug $\mathrm{BH}$ was loaded into the BC/PAni composite hydrogel through simple diffusion method. Briefly, equally cut pieces of BC/PAni hydrogel were added into $0.5 \%(\mathrm{w} / \mathrm{v}) \mathrm{BH}$ solution prepared in PBS (pH 7.4) and stirred at $150 \mathrm{rpm}$ for $30 \mathrm{~min}$. The $\mathrm{BC} / \mathrm{PAni}$ hydrogels loaded with $\mathrm{BH}$ were removed from the solution and washed three times with distilled water to remove excess or surface adsorbed $\mathrm{BH}$.

\subsection{Characterization of $\mathrm{BC}, \mathrm{BH}, \mathrm{BC} / \mathrm{PAni}$, and BC/PAni/BH hydrogel}

The synthesized BC/PAni electroactive hydrogels before and after loading the $\mathrm{BH}$ were characterized for various features through different techniques. The thickness of the layers in hydrogel was measured by micrometer in the wet state. The surface morphology of freeze-dried samples was observed through FE-SEM. Briefly, the samples were fixed on a brass holder and coated with gold on a $\mathrm{Cu}$ SEM disk and analyzed through a Nova Nano SEM450 FE-SEM (FEI, Holland). FTIR spectra of the freeze-dried samples were recorded using a Vertex FTIR spectrophotometer (Germany; spectral range 4000-400 $\mathrm{cm}^{-1}$; beam splitter: Ge-coated on $\mathrm{KBr}$; detector: DTGS; resolution: $0.25 \mathrm{~cm}^{-1}$ (step selectable)) to determine the chemical structure of hydrogels and possible interactions between the components. The obtained IR data was transferred to PC to acquire spectra. The cyclic voltammetry measurements were carried out at room temperature with $\mathrm{CHI} 660 \mathrm{D}$ electrochemical station $(\mathrm{CHI}$ Instrument) in the potential range of $-1.2 \mathrm{~V}$ to $1.2 \mathrm{~V}$ and a series of scan rate $(25,50,75$ and $100 \mathrm{mV} / \mathrm{s})$ were measured. Measurements on the hydrogel were carried out between two $\mathrm{SnO}_{2} \mathrm{~F}$ coated glass electrodes. The BC/PAni hydrogel was immersed in PBS buffer solution at different $\mathrm{pH}$ values ranging from 1.7 to 14.5 to study its electrical properties.

\subsection{Drug release behavior of $\mathrm{BC} / \mathrm{PAni}$ hydrogel}




\section{under applied voltage at different $\mathbf{p H}$}

Different $\mathrm{pH}$ and voltages $(0-0.5 \mathrm{~V})$ were applied to determine the drug release behavior of $\mathrm{BC} / \mathrm{PAni}$ hydrogel by measuring the amount of released drug using the gravimetric method. Briefly, a specialized device shown in Fig. 1 was designed where BC/PAni hydrogel was placed in an insulated framework and fixed between two conductive glass electrodes. Several pores $(\mathrm{R}=1 \mathrm{~mm})$ were drilled in one of the conductive glass electrode for the drug release. The $\mathrm{BH}$ loaded $\mathrm{BC} /$ PAni hydrogel was added into $80 \mathrm{~mL}$ PBS solution at $37^{\circ} \mathrm{C}$ and $\mathrm{pH}$ varying from 2.2 to 11.6 . At specified time intervals, a $1.0 \mathrm{~mL}$ PBS solution was taken out from the system for drug release analysis while maintaining its total volume by adding $1.0 \mathrm{~mL}$ of fresh PBS solution. The amount of released $\mathrm{BH}$ was determined after every 1 , $2,4,6,8,12$, and $24 \mathrm{~h}$ for 3 consecutive days using a UV spectrophotometer by measuring the absorbance at $341 \mathrm{~nm}$. The amount of total released drug was determined by a cumulative release curve. The results were presented as cumulative percentage (\%) release as a function of time using the following formula:

$$
\text { Cumulative drug release }(\%)=\frac{\mathrm{W}_{\mathrm{I}}}{\mathrm{W}_{\mathrm{D}}} \times 100 \%
$$

Where $\mathrm{W}_{\mathrm{I}}$ is the amount of cumulative $\mathrm{BH}$ released until a given time while $W_{D}$ is the total amount of loaded $B H$ in the hydrogel.

The release mechanism of $\mathrm{BH}$ from electroactive $\mathrm{BC} / \mathrm{PAni}$ hydrogel was determined using Higuchi order (Eq. 2), Zero order (Eq. 3), and Korseymer-pappas (Eq. 4):

$$
\mathrm{Q}=\mathrm{Q}_{0}+\mathrm{kt}^{\frac{1}{2}}
$$

where $\mathrm{Q}$ is the cumulative amount of released drug at a given time, $\mathrm{k}$ is the Higuchi constant, and $t$ is the drug release time. Higuchi order gives a cumulative percentage of released drug versus square root of time and was used to investigate the drug release from the matrix based on Fickian diffusion.

$$
\mathrm{Q}_{\mathrm{t}}=\mathrm{Q}_{0}+\mathrm{kt}
$$

where $\mathrm{Q}$ is the amount of released drug, $\mathrm{Q}_{0}$ is the initial amount of drug in the solution, and $\mathrm{k}$ is the zero order release constant. Zero order gives cumulative drug release versus time and was used to investigate the concentration-independent drug release rate from the formulation.

$$
\mathrm{F}=\left(\frac{\mathrm{M}_{\mathrm{t}}}{\mathrm{M}}\right)=\frac{\mathrm{M}_{0}}{\mathrm{M}}+\mathrm{kt} \mathrm{t}^{\mathrm{n}}
$$

where $F$ is the fraction of drug released at time ' $t$ ', $M_{0}$ is the burst release at the beginning; $M_{t}$ is the amount of drug released time ' $t$ ', $\mathrm{M}$ is the total loaded drug, ' $\mathrm{k}$ ' is the kinetic constant, and ' $\mathrm{n}$ ' is the release constant. Korsemyer-Peppas gives absolute cumulative amounts of drug release at time ' $t$ '.

\section{Results and discussion}

\subsection{Development and morphological analysis of electroactive BC/PAni hydrogel}

An electroactive hydrogel was developed through a chemical oxidation polymerization of PAni along BC fibers for the development of a controlled drug release system (Fig. 2). During the chemical polymerization, the aniline salt diffused out of the monomer treated BC hydrogel while the APS diffused into the hydrogel network forming two gradients. Thereafter, PAni formation started at the surface along the BC fibers and grew inwards. With the polymerization of PAni and the consumption of the reactant, the pores of hydrogel narrowed and the reaction rate decreased. Thus by controlling the different reaction time, the sandwiched BC/PAni hydrogel with different PAni thickness was achieved.

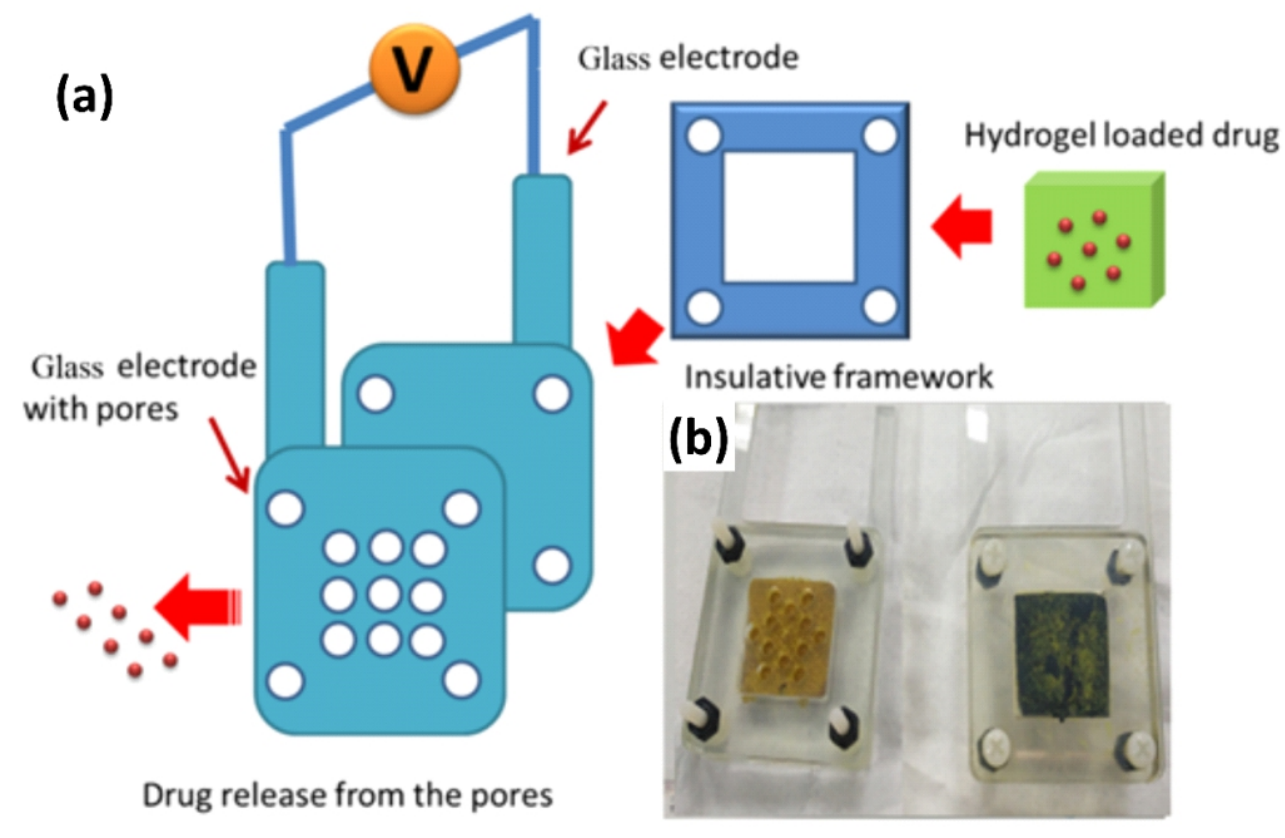

Fig. 1 (a) Schematic illustration and (b) photograph of conductive glass electrode. 

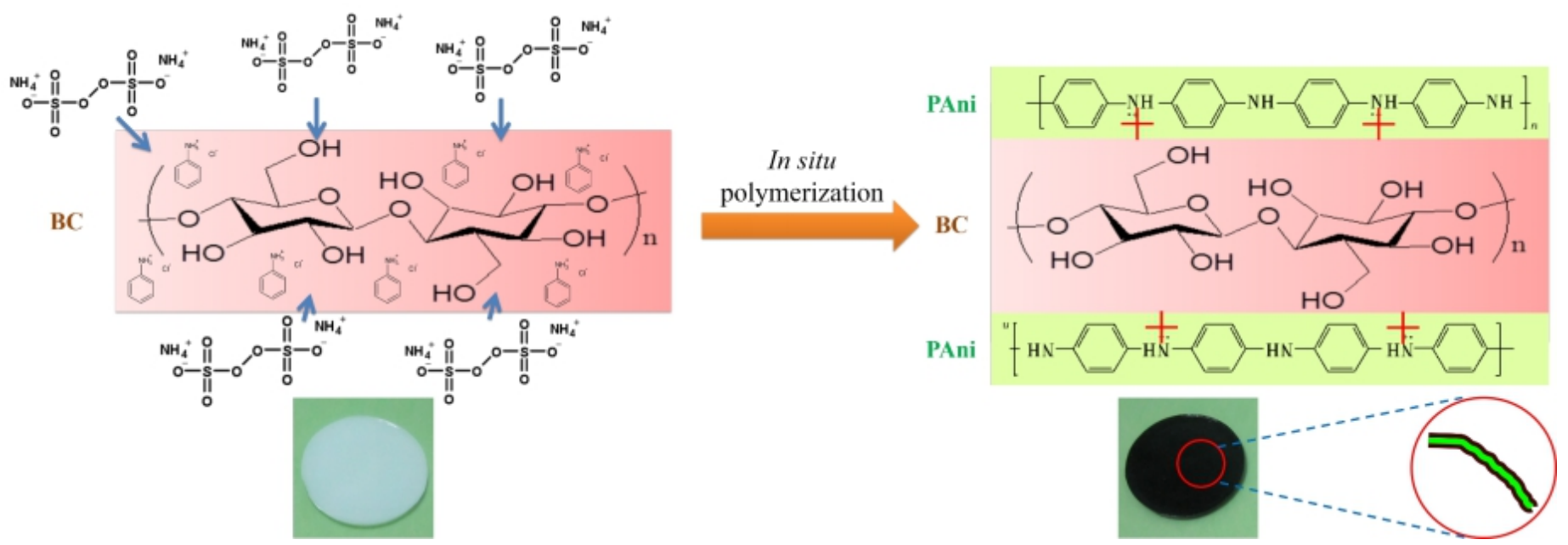

Fig. 2 Schematic illustration of chemical oxidation polymerization of aniline along the BC fibers during the preparation of BC/PAni sandwich hydrogel structure.

The multilayered BC/PAni hydrogel, variation in its thickness, and structural morphology are shown in Fig. 3. The naked-eye observation revealed the synthesis of differently colored five-layered hydrogel containing the top dark and green layers (PAni), middle white layer (BC), and lower green and dark layers (PAni) (Fig. 3a). The thickness analysis revealed that it varied differently for these layers in a time-dependent manner (Fig. 3b). For instance, the thickness of top dark layer increased slightly with time and reached to a maximum of $30 \%$ of the total thickness of hydrogel after 70 min. This indicates the occurrence of polymerization of PAni along $\mathrm{BC}$ fibers present at the top surface. In contrast, the thickness of top green layer was nearly constant after 70 min indicating the uniform polymerization of $\mathrm{BC}$ fibers present here. A similar trend was observed in the lower green and dark layers whose thicknesses were increased and constituted $18 \%$ and $16.5 \%$, respectively of the total thickness of the hydrogel. Interestingly, the thickness of middle layer (a)

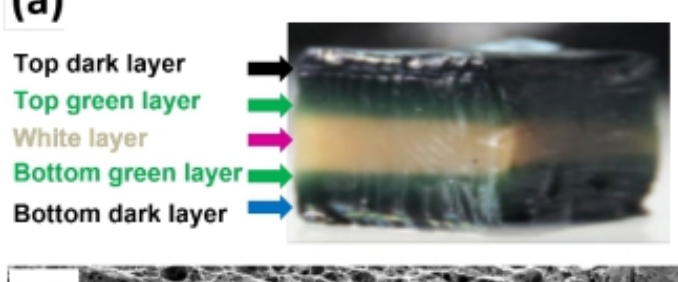

(b)

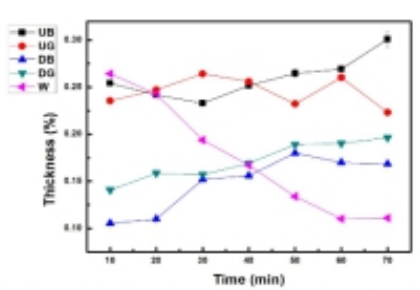

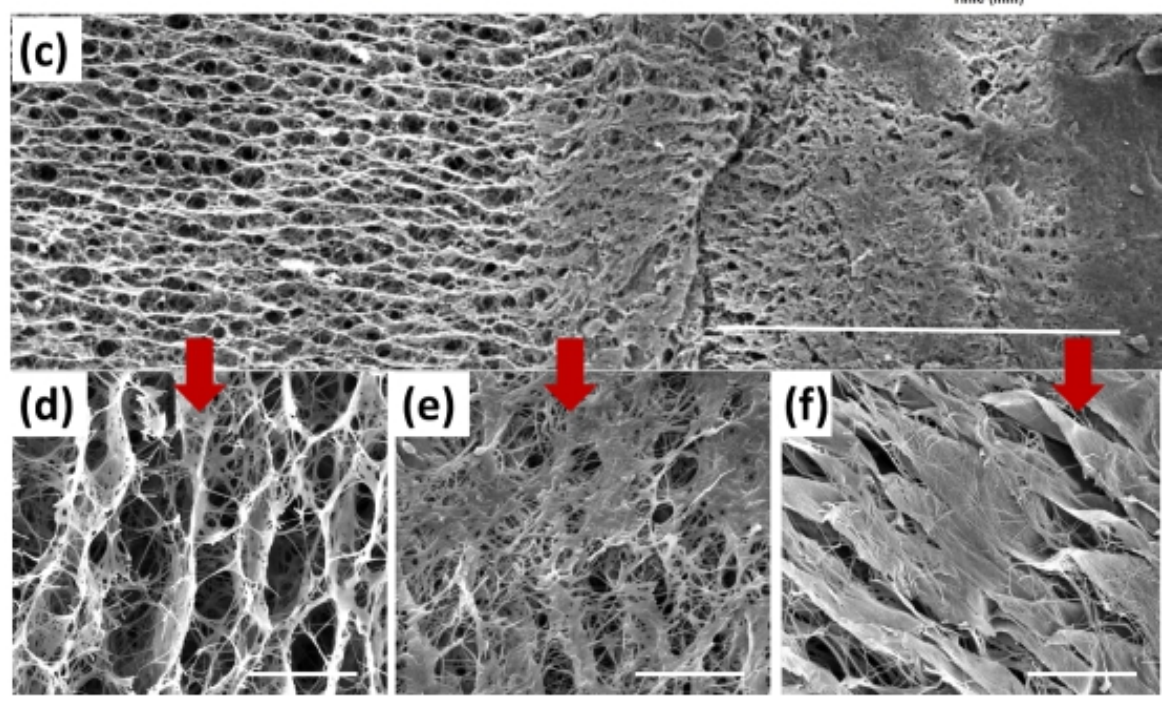

Fig. 3 Illustration of (a) synthesis of a multilayered sandwich structure of BC/PAni hydrogel, (b) thickness-time correlation curves, and SEM micrographs of (c) porous 3D nanostructure of BC/PAni hydrogel, (d) middle white layer, (e) top and bottom green layer, and (f) top and bottom dark layer. The scale bar for (c) is $100 \mu \mathrm{m}$ and (d), (e), and (f) is $10 \mu \mathrm{m}$. 
(BC) was decreased significantly from $26 \%$ to $12 \%$ after $70 \mathrm{~min}$ indicating that polymerization of PAni occurred along the BC fibers. A cross-sectional analysis of electroactive BC/PAni hydrogel via SEM showed a porous 3D nanostructure of BC network (Fig. 3c), which can potentially allow the impregnation of different size and shape materials through physical diffusion or chemical polymerization. The polymerization of PAni along the BC fibers is shown in Fig. 3d-f, which demonstrate that PAni was densely arrayed along BC fibers at the top and the bottom surfaces. Fig. $3 \mathrm{~d}$ shows that $\mathrm{BC}$ fibers were hardly polymerized in the middle layer. In contrast, the top and bottom green layers indicated lower polymerization of PAni along the BC fibers (Fig. 3e) compared to those of the top and the bottom dark layers (Fig. 3f), which indicate that polymerization of PAni along BC fibers took place gradually from outer to inner matrix.

The SEM micrographs were analyzed using Sigma Scan Pro 5 software for determination of pore size in different layers (Table 1). The results showed that compared to non-polymerized BC (middle layer) $(51.54 \%)$, the porosity of BC/PAni hydrogel at the top and bottom surfaces significantly decreased to $12.85 \%$ and $7.72 \%$, respectively which is in accordance with the SEM micrographs. A similar trend was observed for the maximum, minimum, and mean pore sizes of the top, bottom, and middle layers indicating the successful polymerization of PAni and expansion of $\mathrm{BC}$ fibers present at the top and the bottom surfaces of BC/PAni hydrogel.

Table 1. Porosity analysis of different layers in the BC/PAni sandwich hydrogel structure.

\begin{tabular}{lccc}
\hline & Middlewhite & Top-bottom & Top-bottom \\
Property & layer & green layer & dark layer \\
\hline Porosity(\%) & 51.54 & 12.85 & 7.72 \\
Meanporesize(nm) & 42.9 & 26.6 & 15.7 \\
Maximumporesize(nm) & 560 & 141 & 180 \\
Minimumporesize(nm) & 10 & 10 & 6.8 \\
\hline
\end{tabular}

\subsection{FTIR characterization of BC/PAni electroactive hydrogel}

FTIR is an important spectroscopic technique to investigate the presence of different functional groups in a molecule and nature of chemical bonds and functional groups linking them together on the surface a molecule, polymer, or an electroactive hydrogel. ${ }^{26}$ Therefore, FTIR characterizations of $\mathrm{BC}, \mathrm{BH}, \mathrm{BC} / \mathrm{PAni}$, and $\mathrm{BC} / \mathrm{PAni} / \mathrm{BH}$ hydrogel were carried out and their FTIR spectra were comparatively analyzed to verify the basic chemical structures of $\mathrm{BC}$ and $\mathrm{BH}$ and investigate the nature of chemical interactions between $\mathrm{BC}$, PAni, and $\mathrm{BH}$ in the developed $\mathrm{BC} / \mathrm{PAni}$ drug delivery system. The combined spectra of $\mathrm{BC}, \mathrm{BH}, \mathrm{BC} / \mathrm{PAni}$, and $\mathrm{BC} / \mathrm{PAni} / \mathrm{BH}$ hydrogel are shown in Fig. 4, which reveal the characteristic peaks for different functional groups in $\mathrm{BC}$, PAni, and $\mathrm{BH}$ and respective peak shift and intensities after the chemical oxidation polymerization of $\mathrm{BC}$ and PAni and loading of $\mathrm{BH}$ in the $\mathrm{BC} / \mathrm{PAni}$ electroactive hydrogel.

The FTIR spectrum of pure $\mathrm{BC}$ showed characteristic peaks at $3347 \mathrm{~cm}^{-1}$ and $2895 \mathrm{~cm}^{-1}$ due to stretching vibration of $\mathrm{OH}$ and $\mathrm{CH}$ functional groups, respectively. The presence of $\mathrm{CH}$ stretching vibration was further supported by the presence of several peaks between $1450 \mathrm{~cm}^{-1}$ and $1200 \mathrm{~cm}^{-1}$. Further, two additional characteristic peaks at $1443 \mathrm{~cm}^{-1}$ and $1417 \mathrm{~cm}^{-1}$ represent the symmetric deformation and bending vibration of $\mathrm{CH}$ functional group in pure $\mathrm{BC}$. The peak for glucose carbonyl group (CO) appeared at $1673 \mathrm{~cm}^{-1}$. The peak due to C-O-C stretching vibrations in $\mathrm{BC}$ appeared at $1067 \mathrm{~cm}^{-1}$. The FTIR spectrum of BH showed characteristic peaks at $1600 \mathrm{~cm}^{-1}$ and $1500 \mathrm{~cm}^{-1}$ for quaternary iminium ion $(\mathrm{C}=\mathrm{N})$ and stretching vibration of aromatic ring $(\mathrm{C}=\mathrm{C})$, respectively, which is in agreement with a previous study. ${ }^{27}$ Other major absorption bands between $1000 \mathrm{~cm}^{-1}$ and $1116 \mathrm{~cm}^{-1}$ represent free primary amino group $\left(\mathrm{NH}_{2}\right)$. A characteristic peak for $\mathrm{CH}_{3}$ stretching vibration appeared at $1380 \mathrm{~cm}^{-1}$ in the $\mathrm{BH}$ spectrum, which was absent in the FTIR spectrum of pure BC. In the FTIR spectrum of BC/PAni, the intensity of $\mathrm{OH}$ functional group at 3347 $\mathrm{cm}^{-1}$ was significantly reduced, indicating the chemical oxidation polymerization of PAni along the $\mathrm{BC}$ fibers. It was further confirmed by the appearance of two additional strong peaks at $1460 \mathrm{~cm}^{-1}$ and $1560 \mathrm{~cm}^{-1}$, which were assigned to quinone and benzene rings stretching oscillation, respectively, which is in agreement with previous studies. ${ }^{1925}$ In conductive PAni, the quinonoid and benzenoid units are generally present in equal ratio, thus intensities of their respective bands appeared at same position in the FTIR spectrum. ${ }^{28}$ The presence of equal intensity bands for quinonoid and benzenoid units in the FTIR spectrum of BC/PAni indicates that these possess a similar structure to that of the conductive PAni, thus the composite is expected to possess high electrical conductivity. The FTIR spectrum of BC/PAni did not show NH stretching absorption which is in agreement with a previous study ${ }^{25}$ indicating the reduction of $\mathrm{BC}$ via dehydration-condensation with PAni and resulted in disappearance or reduction of $\mathrm{OH}$ and $\mathrm{NH}$ bonds. The FTIR spectrum of BC/PAni/BH showed different absorption bands compared to pure $\mathrm{BC}, \mathrm{BH}, \mathrm{BC}-\mathrm{BH}$, and $\mathrm{BC} / \mathrm{PAni}$ hydrogel. These results suggest successful chemical oxidation polymerization of $\mathrm{BC}$ and PAni and incorporation of $\mathrm{BH}$ into the hydrogel matrix through the synthesis of polyelectrolyte complex between BC and PAni.

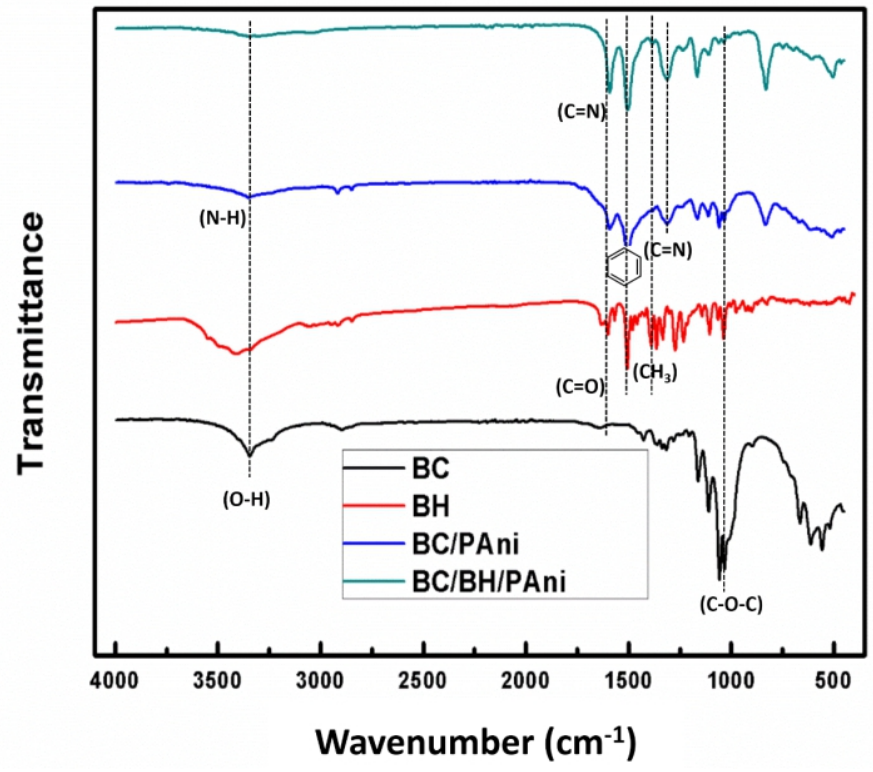

Fig. 4 FTIR spectra of pure $\mathrm{BC}$, drug $\mathrm{BH}, \mathrm{BC} / \mathrm{PAni}$, and drug loaded BC/PAni/BH hydrogel at $\mathrm{pH}$ 7.4. 
(a)

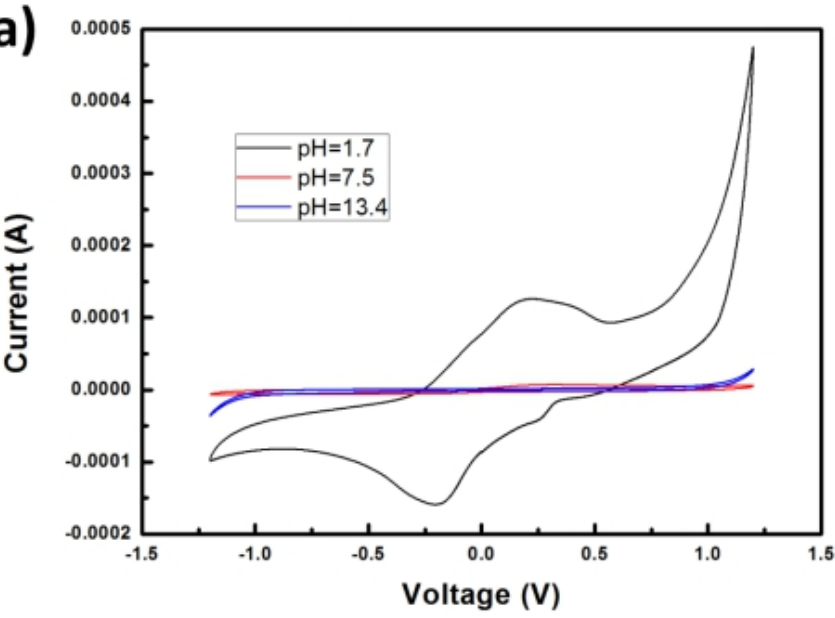

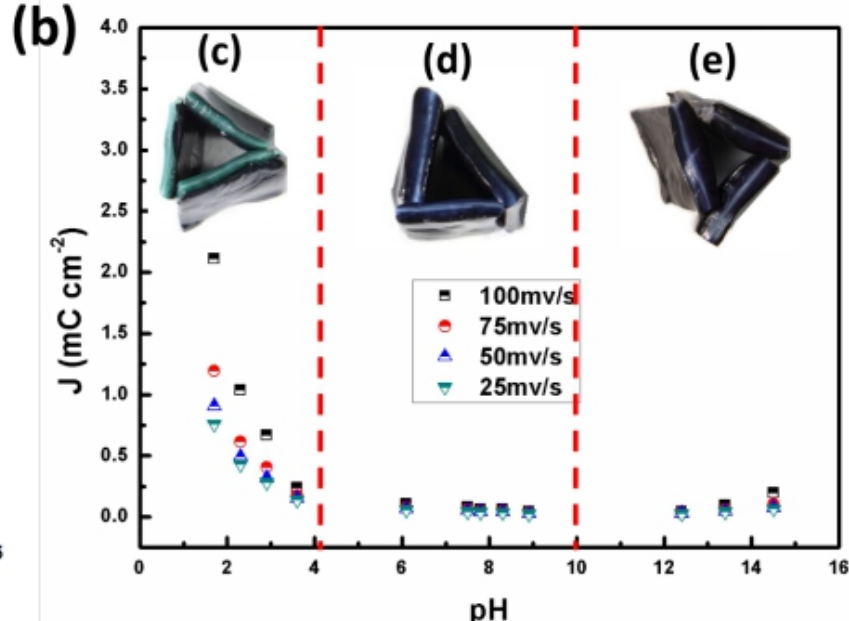

Fig. 5 (a) Cyclic voltammograms curves of BC/PAni at different $\mathrm{pH}$ (1.7, 7.5 and 13.4), (b) surface current density under different pH conditions calculated from cyclic voltammograms, and demonstration of color variations from (c) green to (d, e) dark when environment was changed from acidic to neutral/alkaline.

\subsection{Cyclic voltammogram analysis}

The redox activity determined via cyclic voltammogram (CV) analysis showed that BC/PAni possesses better conductivity in the acidic solution due to doping of proton acid. CV voltammograms of $\mathrm{BC} / \mathrm{PAni}$ hydrogel at different $\mathrm{pH}$ values ranging from 1.7 to 14.5 are shown in Fig. 5a and Figure S1 using which the surface current density $(\mathrm{J})$ was calculated (Table S1 and Fig. 5 b). The results showed that, at $\mathrm{pH}<4$, the doping of proton acid made $\mathrm{BC} / \mathrm{PAni}$ hydrogel more electrochemical because of the formation of radical cations; whereas, the positive charges on the polymer chain are compensated by the imide ions due to the acidic environment. At this stage, PAni was present in the form of emeraldine salt (ES) which showed better conductivity, wherein the $\mathrm{H}^{+}$ions and the substituted cations doped on the polymer chain can transfer electrons under electrical stimulation. This process made the BC/PAni hydrogel to possess high surface current density $(\mathrm{J})\left(2.11 \mathrm{mC} / \mathrm{cm}^{2}\right.$ at scan rates of 100 $\mathrm{mV} \mathrm{s}^{-1}$ when $\mathrm{pH}=1.7$ ). In contrast, at $\mathrm{pH}>4$, the PAni in $\mathrm{BC} /$ PAni hydrogel was translated to eigenstate form due to loss of proton acid; thus decreased the surface current density (J) (Table S1). These results demonstrated that PAni retained its $\mathrm{pH}$ sensitivity even after it was combined with $\mathrm{BC}$ as demonstrated by the color variation from green to dark in acidic to neutral/alkaline conditions (Fig. 3a, b and c) in a reversible way.

\section{4 pH-sensitive controlled release behavior of BH-loaded BC/PAni hydrogels}

The mechanism of $\mathrm{BH}$ release from the electroactive BC/PAni hydrogel was investigated under different physiological media of simulated gastric fluid (SGF) ( $\mathrm{pH}$ 2.2), simulated intestinal fluid (SIF) ( $\mathrm{pH}$ 6.8), and $\mathrm{pH} 11$ (Fig. 6a). In our previous study, BC membranes were used as carrier which demonstrated an extended released time for two model drugs, berberine hydrochloride and berberine sulphate. The results showed up to $80 \%$ release of $\mathrm{BH}$ after $20 \mathrm{~h}, 8 \mathrm{~h}$, and $10 \mathrm{~h}$ at $\mathrm{pH} 2.1,6.8$, and $\mathrm{pH} 12$, respectively. ${ }^{22}$ In the current study, the electroactive BC/PAni hydrogel showed $37 \%$, $45 \%$, and $69 \%$ drug release at $\mathrm{pH} 2.2,6.8$, and 11 , respectively after $59 \mathrm{~h}$ (Fig. 6b). Compared to pure BC hydrogel, the multilayered
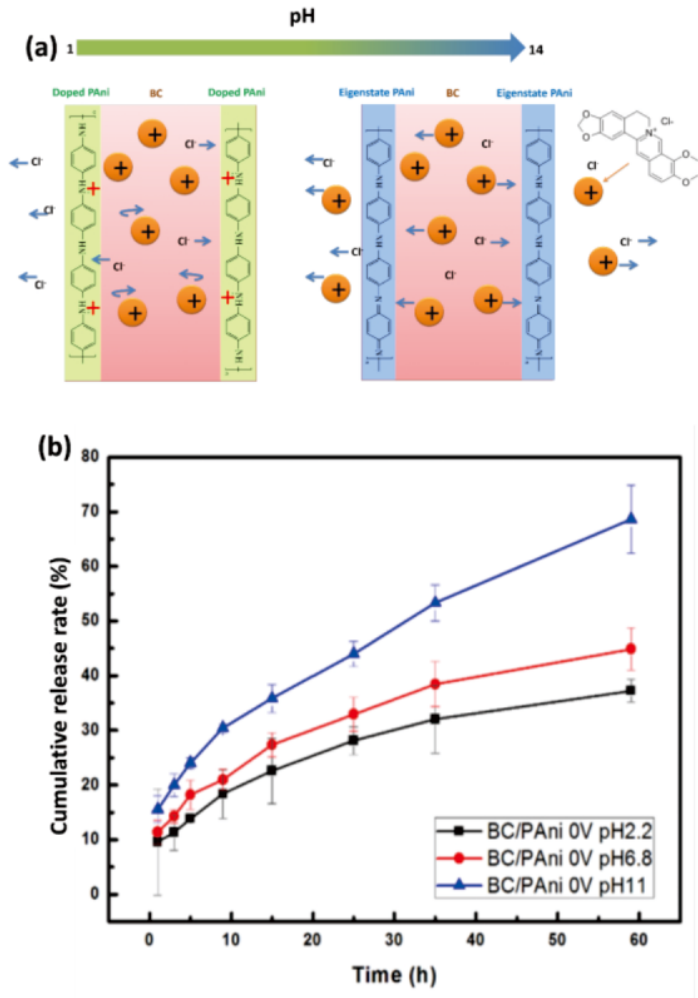

Fig. 6 (a) Illustration of drug release profiles from BC/PAni hydrogels at different $\mathrm{pH}$ values. (b) The cumulative amount of $\mathrm{BH}$ released from $\mathrm{BC} / \mathrm{PAni}$ hydrogels at time ' $\mathrm{t}$ ' (h) under different $\mathrm{pH}$ values at $37^{\circ} \mathrm{C}$.

sandwich microstructure of BC/PAni hydrogel showed an extended drug release profile of $\mathrm{BH}$, which could be attributed to the different porosity, $12.85 \%$ and $7.72 \%$, at the top and the bottom surfaces, which significantly reduced the exchange rate of molecules.

The schematic of drug release behaviors of $\mathrm{BH}$ loaded in $\mathrm{BC} / \mathrm{PAni}$ hydrogels at $\mathrm{pH} 2.2-11$ is shown in Fig. 6a. It was 
observed that $\mathrm{BH}$ was released fast under alkaline conditions and slowly in the acidic environment. At low $\mathrm{pH}$ values $(<4)$, the PAni in $\mathrm{BC} / \mathrm{PAni}$ hydrogels was doped by protonic acid. The outer layer of BC/PAni was positively charged as a smart charge filter to separate ions, and the cations migrated through the network leaving behind anions (berberine ${ }^{+}$) in the $\mathrm{BC}$ network; thereby resulted in an extended anions drug release time. In contrast, at $\mathrm{pH}>4$, the PAni in $\mathrm{BC} / \mathrm{PAni}$ from doped PAni was translated to eigenstate PAni form; thus both cations and anions moved freely across the outer layer of $\mathrm{BC} / \mathrm{PAni}$ hydrogel which resulted in an increased drug release rate. The BC/PAni drug release system showed a typical $\mathrm{pH}$ sensitive release behavior; i.e. fast in alkaline and slow in acid environments. The hydrogels could protect the drug in an acidic environment (SGF) ( $\mathrm{pH}$ 2.2) before releasing it into the small intestine (SIF) ( $\mathrm{pH}$ 6.8) for drug absorption into the blood circulation system.

\subsection{Electro-responsive release behavior of $\mathrm{BH}-$ loaded BC/PAni hydrogels}

The release behavior of $\mathrm{BH}$ from $\mathrm{BC} / \mathrm{PAni}$ hydrogels with and without applied voltage at $\mathrm{pH} 2.2,6.8$, and 11 is shown in Fig. 7. The results showed that $\mathrm{BH}$ released from $\mathrm{BC} / \mathrm{PAni}$ hydrogel after 3 $\mathrm{h}$ at $0 \mathrm{~V}$ was $11 \%, 14 \%$, and $19 \%$ at $\mathrm{pH} 2.2,6.8$, and 11 , respectively. With further increase in time up to $59 \mathrm{~h}$, the $\mathrm{BH}$ released from BC/PAni hydrogel at $0 \mathrm{~V}$ was found to be $37 \%, 44 \%$, and $68 \%$ at $\mathrm{pH} 2.2,6.8$, and 11 , respectively. In contrast, the $\mathrm{BH}$ released from $\mathrm{BC} / \mathrm{PAni}$ hydrogel was increased to $12 \%, 15 \%$, and $22 \%$ at $\mathrm{pH} 2.2,6.8$, and 11 , respectively with voltage $(0.5 \mathrm{~V})$ input. With further increase in time up to $59 \mathrm{~h}$, the $\mathrm{BH}$ released from BC/PAni hydrogel was $44 \%, 47 \%$, and $74 \%$ at $\mathrm{pH} 2.2,6.8$, and 11 , respectively with voltage $(0.5 \mathrm{~V})$ input. These results demonstrated that the released $\mathrm{BH}$ was significantly increased upon voltage input indicating the BC/PAni system under a consideration as an electroactive hydrogel. In the current system, the anions including berberine ${ }^{+}$preferred to escape and moved to cathode while cations migrated to anode upon application of an electrical stimulus. In conclusion, the electrical stimulation accelerated the directed release of ionic drug $\mathrm{BH}$ (Fig. 7a). A comparison of berberine derivative release behavior of current electroactive BH/PAni sandwiched hydrogel with previously reported drug release systems is given in Table 2.

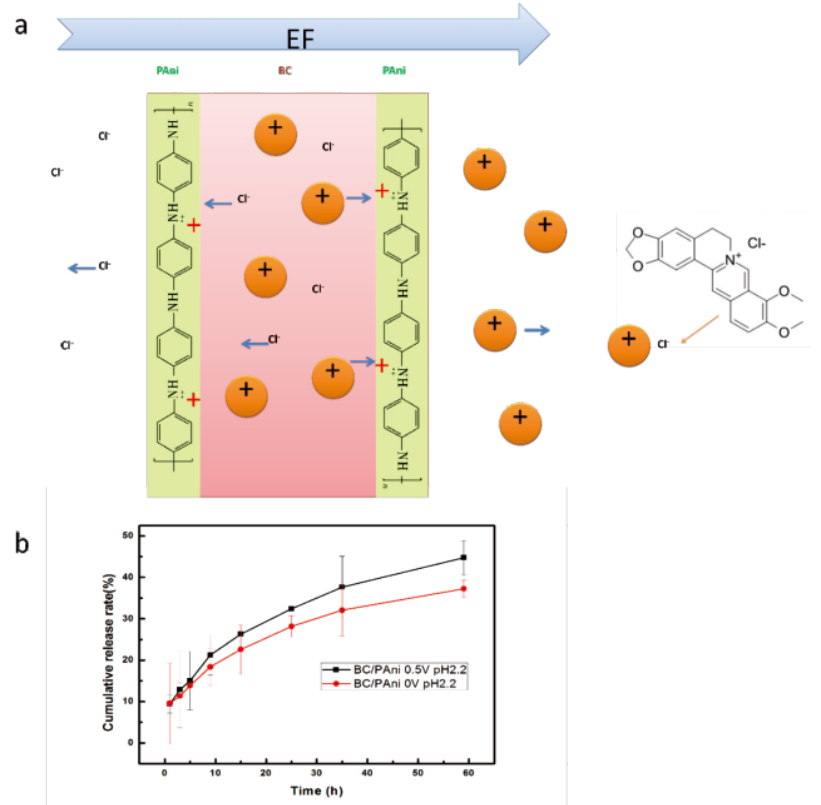

c

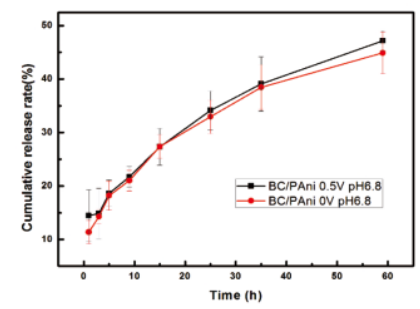

d

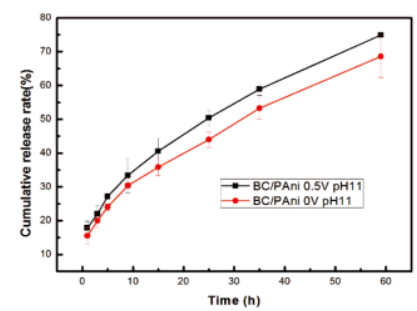

Fig. 7 (a) Illustration of drug release profiles from BC/PAni hydrogels with voltage input. Cumulative release profile of $\mathrm{BH}$ at (b) $\mathrm{pH} 2.2$, (c) $\mathrm{pH} 6.8$, and (d) $\mathrm{pH} 11$ is demonstrated. The release of $\mathrm{BH}$ from $\mathrm{BC} / \mathrm{PAni}$ with and without voltage input over a period of $59 \mathrm{~h}$.

Table 2. Comparison of different drug release systems for berberine derivatives

\begin{tabular}{|c|c|c|c|}
\hline Drug release system & Control method & Release time at $40 \%$ & Ref. \\
\hline Spray-dried muco-adhesive & --- & $<10 \min$ & 29 \\
\hline \multicolumn{4}{|l|}{ micro particles } \\
\hline Self-nanoemulsifying & --- & $10 \mathrm{~min}$ & 30 \\
\hline MgAl hydrotalcite & --- & $20 \mathrm{~min}$ & 31 \\
\hline PEG-lipid-PLGA NPs & $\mathrm{pH}$ & $16 \mathrm{~h}$ & 32 \\
\hline PAMAM & Conjugation & $1-8 \mathrm{~h}$ & 33 \\
\hline Pellets & Pelletsize & $30-90 \mathrm{~min}$ & 34 \\
\hline BC/PAni & $\mathrm{pH}$, voltage & $59 \mathrm{~h}$ & This work \\
\hline
\end{tabular}



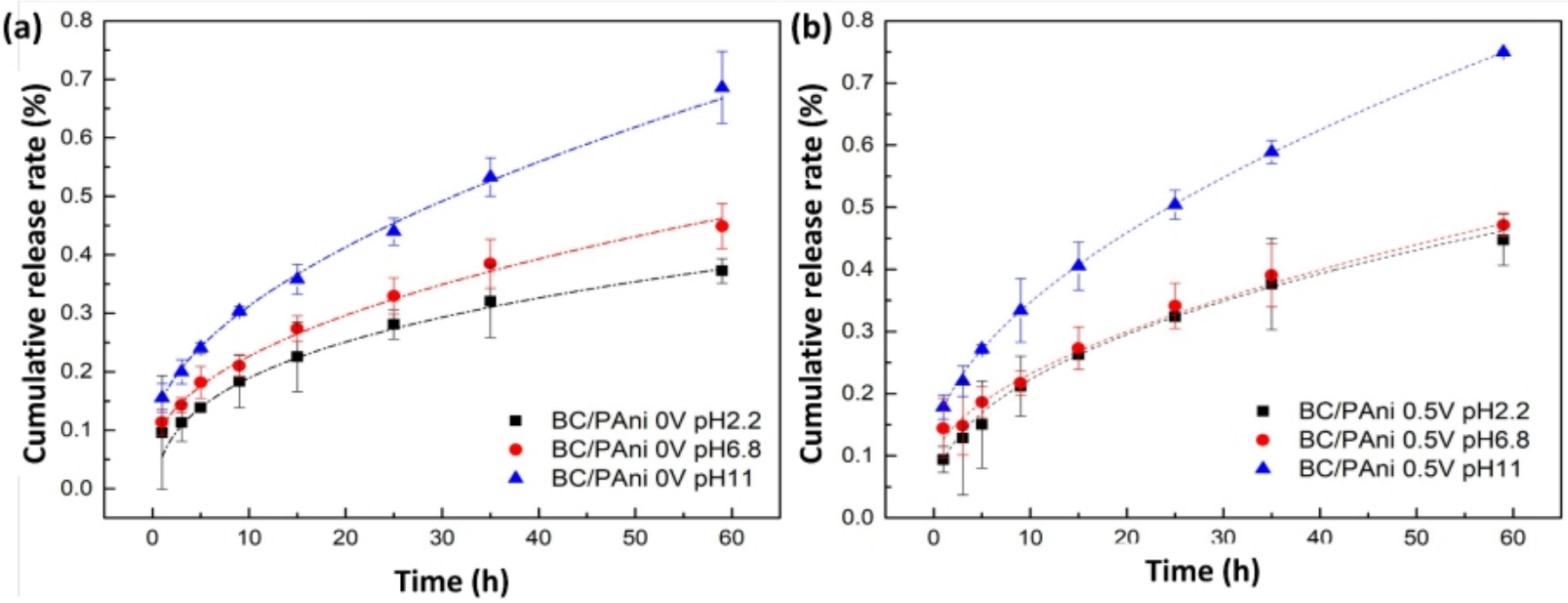

Fig. 8 Korsmeyer-Peppas model for the mechanism of $\mathrm{BH}$ release at different $\mathrm{pH}$ values (a) without (0V) and (b) with voltage $(0.5 \mathrm{~V})$ input at $37^{\circ} \mathrm{C}$.

\subsection{Release kinetic analysis of $\mathrm{BC} /$ PAni hydrogels}

The various release kinetic models developed are supposed to reason different release mechanisms. ${ }^{35,36}$ Therefore, in the current study, the zero-order release equation, Higuchi order equation, and KorsemyerPeppas were used to analyze the in vitro released data. The adjusted correlation coefficients of Zero-order, Higuchi equation, and korsemyer- Peppas kinetic models used in the current study are shown in the supplementary Table S2. The in vitro drug release profiles of $\mathrm{BH}$ at $\mathrm{pH} 2.2,6.8$, and $\mathrm{pH} 11$ were well-fitted with Korsmeyer-Peppas model (Fig. 8). According to this model, a value of $\mathrm{n}<0.5 / 0.45 / 0.43$ for a slab/cylinder/sphere indicates a diffusion controlled drug release. When $\mathrm{n}>1 / 0.89 / 0.85$ for a slab/cylinder/sphere, the drug release behavior is $\mathrm{pH}$-electro sensitive, which is also called case-II transport. In contrast, when $0.5 / 0.45 / 0.43<\mathrm{n}<1 / 0.89 / 0.85$, the drug release behavior is classified as a combination of both phenomenon and is referred to anomalous transport. In the current study, the slab shape samples were prepared with critical n-values as 0.5 and 1 . The results in supplementary Table $\mathrm{S} 3$ suggested that $\mathrm{BH}$ release was more diffusion-based at $\mathrm{pH} 2.2(\mathrm{n}=0.290)$ and 6.8 $(\mathrm{n}=0.464)$ than anomalous type at $\mathrm{pH} 11(\mathrm{n}=0.532)$. In an acidic environment, there were affluent $\mathrm{H}^{+}$ion doping into the PAni which formed a positively charged protective layer, thus these slowed down the diffusion rate of cationic $\mathrm{BH}$ compared to neutral condition at $\mathrm{pH}$ 6.8. At an ambient $\mathrm{pH} \mathrm{11,} \mathrm{the} \mathrm{drug} \mathrm{release} \mathrm{was} \mathrm{rapid} \mathrm{due} \mathrm{to} \mathrm{the}$ precipitation tendency of $\mathrm{BH}$ and $\mathrm{OH}^{-}$. At $\mathrm{pH} 6.8$, the $\mathrm{BH}$ release was more likely to be Fickan diffusion without impeding or driving forces mentioned above. Therefore, BC/PAni hydrogel loaded with cationic drug showed a $\mathrm{pH}$-sensitive release behavior, which can be described as suppressive, normal, and induced while in acidic, neutral, and alkaline ambient environments, respectively.

Upon applied voltage $(0.5 \mathrm{~V})$, the $\mathrm{BH}$ followed diffusion-based release at $\mathrm{pH} 2.2(\mathrm{n}=0.430)$ and anomalous type at $\mathrm{pH} 11(\mathrm{n}=0.561)$; however, it changed to non-Fickan diffusion at $\mathrm{pH} 6.8(\mathrm{n}=0.549)$. The release model change proved that the electrical stimulation played a part during the release process. To be more specific, the voltage input created an electric field between outer BC/PAni layers, which promoted the movement of charged $\mathrm{BH}$ towards the outer layer of hydrogel which in turn increased the $\mathrm{BH}$ release rate, thus the fit exponent $n$-value was increased. The voltage-drive increased release was observed in all experimental groups. At $\mathrm{pH} 2.2$, the exponent ' $n$ ' increased from 0.290 to 0.430 , closed to the critical value which demonstrated that the electrical stimulation possesses the increasing function against acidic force which still took the main influence during the drug release. The release rate was slightly increased at $\mathrm{pH} 11$ which further verified that the voltage input accelerated the whole drug release process even at different $\mathrm{pH}$ values. In summary, the BC/PAni hydrogel could be used as cationic drug carrier, which can show different release rates at different $\mathrm{pH}$ and can promote the drug release under an electrical stimulation.

\section{Conclusions}

A pH-electroactive drug release system was developed through the chemical oxidation polymerization of $\mathrm{BC}$ and PAni for the release of precise quantities of ionic drug. Through a controlled polymerization reaction time, the $\mathrm{BC} / \mathrm{PAni}$ hydrogel system demonstrated multilayered sandwiched structure where PAni was densely arrayed along the $\mathrm{BC}$ fibers; thus resulted in different porosity of BC/PAni hydrogel at the top and bottom surfaces, which significantly extended the drug release time and thus confirmed the proposed hypothesis. Further, the $\mathrm{pH}$-electro sensitive hydrogel demonstrated slow drug release in acid condition and fast release in neutral and alkaline environments. In acidic condition, PAni was doped by protonic acid whereas the outer layer of BC/PAni hydrogel was positively charged as a smart charge filter to separate ions. The cations migrated through the $\mathrm{BC}$ network leaving behind anions (berberine $)^{+}$; thereby extending the anions drug release time. In neutral and alkaline environments, PAni was present in eigenstate form. Both cations and anions moved freely through the outer layers $\mathrm{BC} / \mathrm{PAni}$ which resulted in an increased drug release rate. Upon application of an electrical stimulus, the anions including berberine preferred to escape and moved to cathode while cations migrated to anode. The current system demonstrated rapid and directional drug release with voltage input and demonstrated Korsemyer-Peppas kinetic model with free diffusion. The developed $\mathrm{pH}$-electroactive $\mathrm{BC} / \mathrm{PAni}$ hydrogel with different porosities at the top and the bottom layers can find potential applications in the development of a 
controlled drug system.

\section{Conflict of interest}

There are no conflicts to declare.

\section{Acknowledgements}

This work was supported by National Natural Science Foundation of China (51603079, 31270150, 21774039), China Postdoctoral Science Foundation (2015M572132, 2016M602291), the Doctoral Program of Higher Education of China (20070487096), Open Research Fund of State the Key Scientific Research Foundation of Huazhong University of Science and Technology (HUST-SRF no.0125170002), the Fundamental Research Funds for the Central Universities (HUST2014XJGH009), and the Science and Technology Support Plan in Jiangsu Province of China (BE2014684).

\section{References}

1. F. H. Meng, Z. Y. Zhong and J. Feijen, Stimuli-Responsive Polym. Program. Drug Deliv., 2009, 10, 197-209.

2. Z. Shi, X. Gao, M. W. Ullah, S. Li, Q. Wang and G. Yang, Biomaterials, 2016, 111, 40-54.

3. S. Mura, J. Nicolas and P. Couvreur, Nat. Mater, 2013, 12, 991-1003.

4. A. Guiseppi-Elie, Biomaterials, 2010, 31, 2701-2716.

5. D. Bin Jiang, B. Y. Zhang, T. X. Zheng, Y. X. Zhang and X. Xu, Mater. Lett., 215, 23-26.

6. S. Zhu, L. Li, J. Liu, H. Wang, T. Wang, Y. Zhang, L. Zhang, R. S. Ruoff and F. Dong, ACS Nano, 12, 1033-1042.

7. K. Kontturi, P. Pentti and G. Sundholm, J. Electroanal. Chem., 1998, 453, 231-238.

8. L. M. Lira and S. I. Córdoba De Torresi, Electrochem. commun., 2005, 7, 717-723.

9. M. R. Abidian, D. H. Kim and D. C. Martin, Adv. Mater., 2006, 18, 405-409.

10.M. W. Ullah, M. Ul Islam, S. Khan, N. Shah and J. K. Park, Korean J. Chem. Eng., 34, 1591-1599.

11.Z. Shi, Y. Zhang, G. O. Phillips and G. Yang, Food Hydrocoll., 2014, 35, 539-545.

12.M. W. Ullah, M. Ul-Islam, S. Khan, Y. Kim and J. K. Park, Carbohydr. Polym., 2015, 132, 286-294.

13.M. U. Islam, M. W. Ullah, S. Khan, N. Shah and J. K. Park, Int. J. Biol. Macromol., 2017, 102, 1166-1173.

14.Z. Shi, X. Shi, M. W. Ullah, S. Li, V. V. Revin and G. Yang, $A d v$.
Compos. Hybrid Mater., 1, 79-93.

15.Z. Di, Z. Shi, M. W. Ullah, S. Li and G. Yang, Int. J. Biol. Macromol., 105, 1638-644.

16.L. Fu, J. Zhang and G. Yang, Carbohydr. Polym., 92, 1432-1442.

17.Y. Li, K. Jiang, J. Feng, J. Liu, R. Huang, Z. Chen, J. Yang, Z. Dai, Y. Chen, N. Wang, W. Zhang, W. Zheng, G. Yang and X. Jiang, Adv. Healthc. Mater, 6, 1601343.

18.J. Yang, L. Wang, W. Zhang, Z. Sun, Y. Li, M. Yang, D. Zeng, B. Peng, W. Zheng, X. Jiang and G. Yang, Small, 14, 1702582.

19.A. Jasim, M. W. Ullah, Z. Shi, X. Lin and G. Yang, Carbohydr. Polym., 2017, 163, 62-69.

20.B. Zhang, J. Zhou, S. Li, X. Zhang, D. Huang, Y. He, M. Wang, G. Yang and Y. Shen, Talanta, 131, 243-248.

21.M. Ul-Islam, S. Khan, M. W. Ullah and J. K. Park, Biotechnol. J., 2015, 10, 1847-1861.

22.L. Huang, X. Chen, T. X. Nguyen, H. Tang, L. Zhang and G. Yang, J. Mater. Chem. B, 1, 2976-2984.

23.Z. Shi, Y. Li, X. Chen, H. Han and G. Yang, Nanoscale, 2014, 6, 970-977.

24.M. Ul-Islam, W. A. Khattak, M. W. Ullah, S. Khan and J. K. Park, Cellulose, 2014, 21, 433-447.

25.Z. Shi, S. Zang, F. Jiang, L. Huang, D. Lu, Y. Ma and G. Yang, RSC Adv., 2012, 2, 1040-1046.

26.M. W. Ullah, M. Ul-Islam, S. Khan, Y. Kim and J. K. Park, Carbohydr. Polym., 136, 908-916.

27.S. Ifuku, M. Tsuji, M. Morimoto, H. Saimoto and H. Yano, Biomacromolecules, 2009, 10, 2714-2717.

28.D. W. Hatchett, M. Josowicz and J. Janata, J. Phys. Chem. B, 103, 10992-10998.

29.C. Godugu, A. R. Patel, R. Doddapaneni, J. Somagoni and M. Singh, PLoS One, 9, e89919.

30.Z. Ke, Z.-P. Zhu, Z.-Y. Xu, C. Fang and S.-Q. Hu, Trop J Pharm Res Trop. J. Pharm. Res. May J. Cit. ReportsScience Ed., 14, 747-752.

31.M. A. Djebbi, Z. Bouaziz, A. Elabed, M. Sadiki, S. Elabed, P. Namour, N. Jaffrezic-Renault and A. B. H. Amara, Int. J. Pharm., 506, 438-448.

32.F. Yu, M. Ao, X. Zheng, N. Li, J. Xia, Y. Li, D. Li, Z. Hou, Z. Qi and X. D. Chen, Drug Deliv., 24, 825-833.

33.L. Gupta, A. K. Sharma, A. Gothwal, M. S. Khan, M. P. Khinchi, A. Qayum, S. K. Singh and U. Gupta, Int. J. Pharm., 528, 88-99.

34.Y. Gao, X. Jin, Y. Sun, F. F. Xu and M. Zhang, Adv. Powder Technol., 29, 682-691.

35.S. Dash, P. N. Murthy, L. Nath and P. Chowdhury, Acta Pol. Pharm., 67, 217-223.

36.P. C. Wu, M. J. Tsai, Y. Bin Huang, J. S. Chang and Y. H. Tsai, Int. J. Pharm., 2002, 243, 119-124. 